\section{Decreased threshold of aggregation to low-dose epinephrine is evidence of platelet hyperaggregability in patients with thrombosis}

\author{
Chelsea Hayes, Sumire Kitahara, \\ Oxana Tcherniantchouk \\ Department of Pathology and Laboratory \\ Medicine, Cedars-Sinai Medical Center, \\ Los Angeles, CA, USA
}

\section{Abstract}

Sticky platelet syndrome has been described as a hereditary thrombophilic condition. The aim of this study is to identify the presence of platelet hyperaggregability in patients who have experienced thrombosis. Light-transmittance platelet aggregometry was used to assess for spontaneous platelet aggregation, aggregation in response to full and low-dose (LD) epinephrine (Epi) and adenosine diphosphate, as well as arachidonic acid, and identify a distinct pattern of platelet hyperaggregability. Lighttransmittance platelet aggregometry results were correlated with PFA-100@ (DadeBehring, Marburg, Germany) results, when available. An exaggerated response to LD Epi was found in $68 \%$ of patients with thrombosis compared to only $36 \%$ of healthy controls $(\mathrm{P}=0.034)$. Patients with thrombosis, either arterial or venous, demonstrated an exaggerated response to LD Epi nearly twice as frequently as healthy controls, even without significant family history of thrombophilia or other known risk factors for thrombosis. This suggests that platelet hyperaggregability may be multifactorial in nature and not necessarily hereditary.

\section{Introduction}

Platelet hyperaggregability, or sticky platelet syndrome (SPS), has only been described in a few studies, which mainly consist of case reports without sufficient patient numbers. The lack of a standardized definition has hindered the recognition of platelet hyperaggregability as an important risk factor for thrombosis. While platelet aggregometry (LTA) is considered the gold standard assay to detect qualitative platelet hypofunction, at our institution, a LTA panel is also used to evaluate increased platelet reactivity. However, little data is available to support the utility of such a panel in the evaluation of hyperaggregable platelets.

Prior studies have described sticky platelet syndrome as a congenital platelet abnormality characterized by clinical and laboratory features, such as hyperaggregability in response to varying concentrations of adenosine diphosphate (ADP) and/or epinephrine (Epi). ${ }^{1-5}$ Three aggregation patterns have been associated with SPS: Type I, hyperaggregability to Epi and ADP; Type II, hyperaggregability to Epi alone, ${ }^{1,3,5-7}$ and Type III, hyperaggregability to ADP alone.2,5 These diagnostic criteria are commonly accepted and are used in the majority of published studies available. Additionally, preceding studies identified subsets of healthy volunteers (14-34\%) who appeared to demonstrate a hyperaggregable platelet phenotype, most frequently observed in response to low concentrations of Epi; 8 -10 however, follow-up data and assessment of thrombosis formation in this supposedly hyperaggregable population subset has not been well evaluated.

Despite these data, the mechanism responsible for platelet hyperaggregability and its role in thrombogenesis remains controversial and specific treatment recommendations for patients who exhibit high platelet reactivity, especially in the presence of anti-platelet therapy, are not established. The aim of this study is to use LTA to assess for spontaneous platelet aggregation, aggregation in response to full and low-dose Epi and ADP, as well as aggregation in response to arachidonic acid (AA), and identify a distinct pattern of platelet aggregation in patients without known risk factors who have experienced thrombosis. Additionally, LTA results will be correlated with PFA-100® (Dade-Behring, Marburg, Germany) closure time, when available.

\section{Materials and Methods}

\section{Patient sample}

The study protocol was approved by the institutional review board. All patients who received LTA to assess platelet hyperaggregability from 2006 to 2011 at Cedars-Sinai Medical Center were included in this retrospective investigation $(n=130)$. The history of thrombosis was confirmed using thorough review of institutional medical records, responses to a LTA patient questionnaire submitted with the specimen requisition, and/or corresponding imaging studies. The LTA patient questionnaire also documented family history of thrombosis. Exclusion criteria included history of bleeding, myeloproliferative neoplasms or known coagulation disorders (e.g. Factor V Leiden). As part of the institutional hyperaggregable LTA panel, spontaneous aggregation, aggregation in response to full and LD Epi and ADP, as well as aggregation in response to $\mathrm{AA}$, were measured. Of note, many studies were collected from outpatient
Correspondence: Oxana Tcherniantchouk, Department of Pathology and Laboratory Medicine, Cedars-Sinai Medical Center, 8700 Beverly Blvd., Los Angeles, CA, 90048, USA.

Tel.: +1.310.423.5471 - Fax: +1.310.423.0483

E-mail: oxana.tcherniantchouk@cshs.org

Key words: light-transmittance platelet aggregometry, hyperaggregable platelets, thrombosis, sticky platelet syndrome.

Acknowledgments: the abstract was submitted, accepted and presented at 2012 International Congress on Thrombosis in Nice, France.

Contributions: $\mathrm{CH}$, was involved in data collection, literature search, data analysis and wrote the manuscript; SK, designed the study, analyzed the data and revised the manuscript; OT conceived and supervised the project, designed the study, analyzed the data, revised the manuscript and approved the final version.

Conflict of interests: the authors declare no potential conflict of interests.

Received for publication: 27 January 2014.

Revision received: 13 June 2014.

Accepted for publication: 1 August 2014.

This work is licensed under a Creative Commons Attribution NonCommercial 3.0 License (CC BYNC 3.0).

(C) Copyright C. Hayes et al., 2014

Licensee PAGEPress, Italy

Hematology Reports 2014; 6:5326

doi:10.4081/hr.2014.5326

samples and LTA analysis occurred at least a few weeks after presentation of the thrombotic event. Additionally, LTA was performed on 14 healthy volunteers who were included in this investigation to be used as a pilot control group.

\section{Platelet aggregometry}

The patient and volunteer whole blood specimens were drawn into sodium citrate (3.2\%) and transported at ambient temperature (approximately $20^{\circ} \mathrm{C}$ ) to the laboratory. Platelet-rich plasma (PRP) was prepared by centrifugation at $1000 \mathrm{rpm}$ for 10 minutes and platelet-poor plasma (PPP) was prepared by recentrifugation at $3000 \mathrm{rpm}$ for 15 minutes. The platelet count was adjusted to between $200 \times 10^{3} / \mu \mathrm{L}$ and $300 \times 10^{3} / \mu \mathrm{L}$ with autologous PPP. Platelet aggregation was performed using a BioData-PAP-4 aggregometer (Bio/Data Corp., PA, USA). Platelet aggregation in response to stimulation with the following agonists at doses recommended by the manufacturer, was determined: $500 \mu \mathrm{g} / \mathrm{mL}$ of AA, $20 \mu \mathrm{M}$ of ADP, $2 \mu \mathrm{M}$ of ADP, $100 \mu \mathrm{M}$ of Epi, $10 \mu \mathrm{M}$ of Epi, and with no agonist (i.e. spontaneous) 
(Bio/Data Corp., PA, USA). Low-dose concentrations were 1:10 dilutions of the original concentration. Test procedure: $0.45 \mathrm{~mL}$ PRP was transferred into a cuvette incubated at $37^{\circ} \mathrm{C}$ for 3 minutes. Then $0.05 \mathrm{~mL}$ of the agonist was added into the PRP and the aggregation pattern was allowed to generate for 10 minutes. For spontaneous aggregation, $0.5 \mathrm{~mL}$ PRP was placed into a cuvette without the addition of an aggregation agent. All testing was completed within four hours from the time the sample was obtained.

\section{Values used for interpretation of platelet aggregometry}

Greater than 60\% aggregation was considered normal for full-dose ADP, full-dose Epi and AA (locally determined response cut-off that corresponded with manufacturer's normal ranges provided in the package insert).

Findings suggestive of aspirin effect included one wave of Epi aggregation, ADP disaggregation or a decreased AA response. Aggregation in response to AA of less than 20\% was considered to be adequate aspirin effect. ${ }^{11}$

LD Epi and ADP aggregation was considered exaggerated if greater than $18 \%$, a cut-off determined after discussion with the manufacturer.

Based on institutional experience, spontaneous aggregation was considered exaggerated if greater than $10 \%$.

\section{PFA-100}

PFA-100 (Dade Behring, Marburg, Germany) was performed on the patient samples as per the manufacturer's guidelines. Whole blood was collected into sodium citrate (3.2\%) and $800 \mu \mathrm{L}$ of sample was pipetted into a collagen-Epi cartridge (a unit containing a membrane coated with $2 \mu \mathrm{g}$ of equine Type 1 collagen and $10 \mu \mathrm{g}$ Epi bitartrate) and a collagen-ADP cartridge (a unit containing a membrane coated with $2 \mu \mathrm{g}$ of equine Type 1 collagen and $50 \mu \mathrm{g} \mathrm{ADP}$ ) and loaded into the PFA100 instrument. Using locally determined response cut-offs, the reference ranges are 65185 seconds (s) for collagen-Epi and 71-118s for collagen-ADP, values similar to those suggested by the manufacturer. Therefore, a PFAEpi greater than $185 \mathrm{~s}$ and a PFA-ADP greater than $118 \mathrm{~s}$ was considered prolonged.

\section{Platelet morphology}

LTA results were also correlated with blood smear platelet morphology, when available. Platelet morphology was evaluated using a Wright-Giemsa stained peripheral blood smear.

\section{Statistical methods}

Continuous variables were compared by means of t-test. Categorical variable were compared using the Fisher's exact test. Bivariate
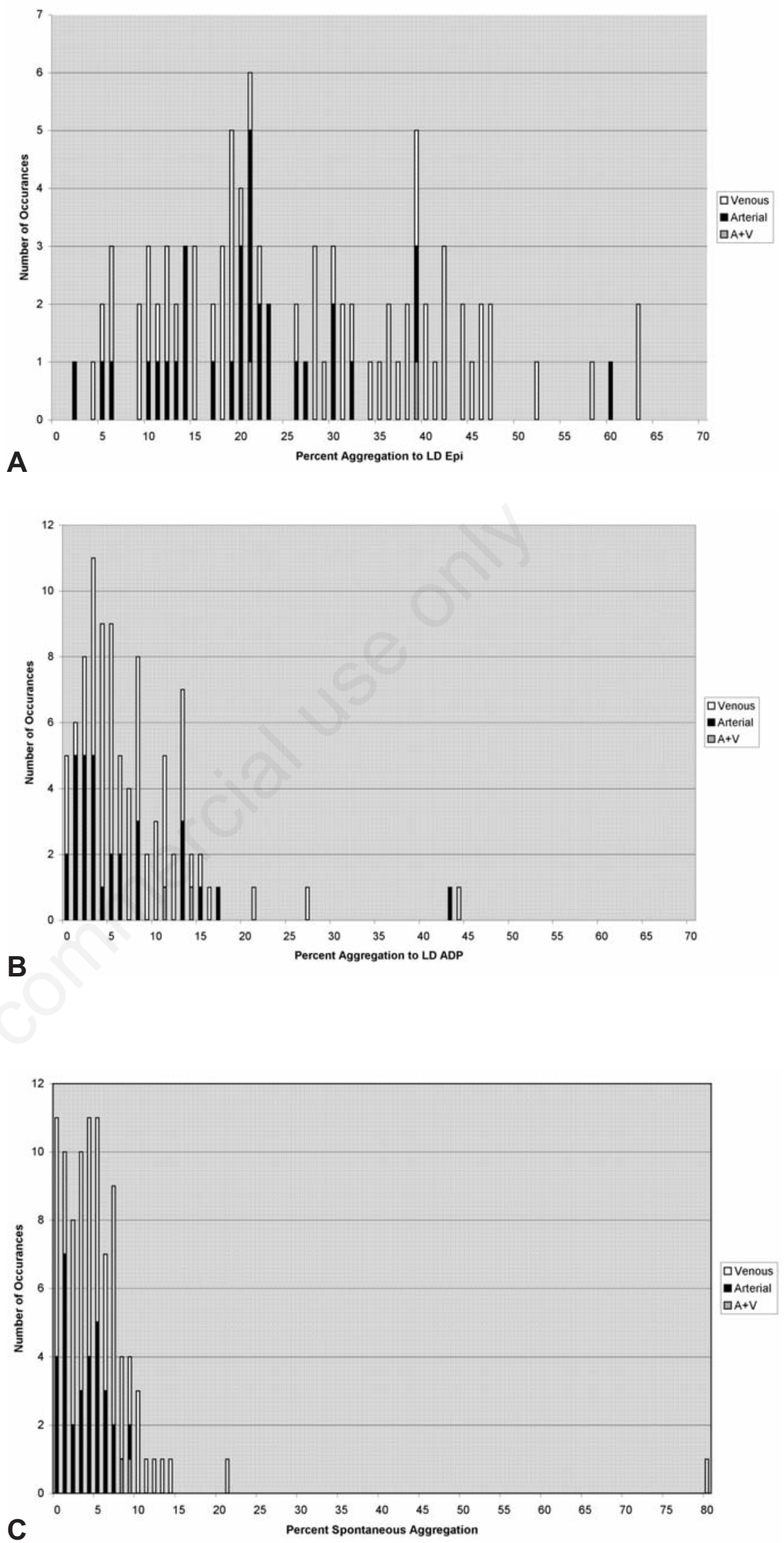

Figure 1. Distribution of aggregation response to: A) low-dose epinephrine; B) low-dose adenosine diphosphate; $\mathrm{C}$ ) no agonist (i.e. spontaneous). 
correlations were determined by calculating the Pearson's correlation coefficient. Statistical analyses were performed using 2013 GraphPad Software ${ }^{\circledR}$ (GraphPad Software, La Jolla, CA, USA).

\section{Results}

\section{Patient sample}

A total of 130 LTA studies were evaluated in this investigation. After application of exclusion criteria, 115 studies remained, with an additional 14 healthy volunteer controls. History of thrombosis was documented in 94 (82\%) studies. Baseline characteristics of patients with thrombosis are shown in Table 1. A relatively high prevalence of venous thrombotic events (65\%) was observed within the patient population, as compared to arterial events (33\%). Additionally, two patients (2\%) had a history of both arterial and venous thromboses (DVT and MI). Documented use of antiplatelet medication at the time of testing was present in 35 (37\%) patients. Of these, 21 reported aspirin use, 6 reported Plavix (Clopidogrel) use and 8 reported use of both aspirin and Plavix (Clopidogrel). Positive family history of thrombosis was indicated in 1 of 115 patients.

\section{Platelet aggregometry results}

An exaggerated response to LD Epi was observed in 64 of 94 patients (68\%) with thrombosis (Figure 1A), while an exaggerated response to LD Epi was observed in only 5 healthy volunteers $(36 \%)(\mathrm{P}=0.034)$. This exaggerated response to LD Epi was present in $65 \%$ and $69 \%$ of patients with arterial and venous events, respectively. Two patients with concurrent arterial and venous events showed abnormal LD Epi results. After exclusion of all patients with either ASA or Plavix effect on LTA, 49 studies remained. Of these, 34 (69\%) showed an exaggerated response to LD Epi. Figure 2 demonstrates an exaggerated LTA response to LD Epi in a patient who developed a deep vein thrombosis while on aspirin.

Abnormalities of platelet aggregation in response to LD ADP (Figure 1B) occurred less frequently and were not found to be significantly different between the two groups $(\mathrm{P}=1.00)$. Spontaneous aggregation was observed in $6(6 \%)$ patients with thrombosis (Figure 1C).

\section{Platelet count and morphology}

No significant difference in platelet count or platelet morphology was identified between patients with thrombosis and healthy controls (Table 2).

\section{PFA-100 results}

LTA results were correlated with PFA-100 results, when available. PFA-Epi and PFA-ADP
Table 1. Characteristics of patients with thrombosis.

\begin{tabular}{lc} 
Characteristics & Patients with thrombosis ( $1=94)$ \\
Age (years), mean \pm SD & $54 \pm 18$ \\
Gender, n (\%) & $44(47)$ \\
Male & $50(53)$ \\
Female & $31(33)$ \\
\hline Documented arterial event, n (\%) & $1(1)$ \\
MI & $25(27)$ \\
TIA or stroke & $5(5)$ \\
Other (ischemic colitis) & $61(65)$ \\
Documented venous event, n (\%) & $37(39)$ \\
DVT & $8(9)$ \\
PE & $13(14)$ \\
DVT and PE & $3(3)$ \\
Other (splanchnic venous thrombosis) & $2(2)$ \\
\hline Arterial and venous event (concurrent DVT and MI), n (\%) & $35(37)$ \\
Documented antiplatelet medication (ASA or Clopidogrel), n (\%) & \\
\hline ASA, aspirin; DVT, deep vein thrombosis; MI, myocardial infarction; n, number; PE, pulmonary thromboembolism; TIA, transient ischemic attack.
\end{tabular}

Table 2. Platelet count and platelet aggregometry results in patients with thrombosis and healthy controls.

\begin{tabular}{lccc} 
& $\begin{array}{c}\text { Patients with } \\
\text { thrombosis }(\mathrm{n}=94)\end{array}$ & $\begin{array}{c}\text { Healthy controls } \\
(\mathrm{n}=14)\end{array}$ & $\mathrm{P}$ \\
Platelet count $\left(\times 10^{3}\right)$, mean $\pm \mathrm{SD}$ & $280 \pm 135$ & $249 \pm 47$ & 0.4 \\
Aggregation $(\%)$, mean \pm SD & & & \\
LD Epi & $26.3 \pm 14.3$ & $21.3 \pm 12.7$ & 0.22 \\
LD ADP & $7.5 \pm 7.4$ & $5.3 \pm 3.2$ & 0.28 \\
Spontaneous & $5.4 \pm 8.6$ & $3.2 \pm 1.5$ & 0.42 \\
\hline Number of Abnormal LTA results, $\mathrm{n}(\%)$ & & & \\
LD Epi & $64(68)$ & $5(36)$ & 0.034 \\
LD ADP & $4(4)$ & $0(0)$ & 1.00 \\
\hline
\end{tabular}

LD ADP, low-dose adenosine diphosphate; LD Epi, low-dose epinephrine; n, number; LTA, platelet aggregometry; SD, standard deviation.
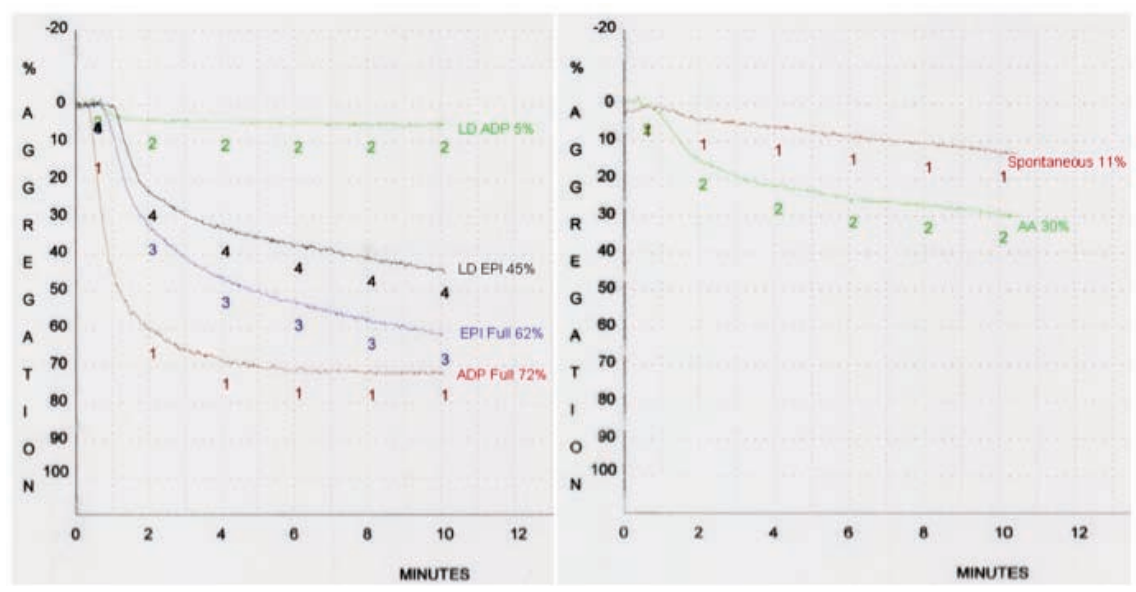

Figure 2. A 69-year-old male presenting with acute deep vein thrombosis of the left lower extremity while on $81 \mathrm{mg}$ of aspirin. Platelet aggregometry remarkable for decreased arachidonic acid aggregation (right graph, curve 2), as well as decreased threshold for aggregation with low-dose epinephrine (left graph, curve 4). 
were available on 88 (94\%) and 85 (90\%) patients with thrombosis, respectively. There was no correlation between the percent aggregation in response to LD Epi and the PFA-Epi $(\mathrm{r}=-0.171)$ or PFA-ADP $(\mathrm{r}=0.096)$ closure times.

\section{Aspirin effect}

Aspirin effect by LTA (defined as $\leq 20 \%$ aggregation with AA) ${ }^{11}$ was noted in 31 of 94 (33\%) patients with thrombosis.

Among the 29 patients who had PFA-100 results available, 11 (38\%) showed a prolonged PFA-Epi closure time, consistent with aspirin effect (i.e. concordant LTA and PFA-100), while the remaining 18 (62\%) showed a normal PFAEpi closure time, suggesting no aspirin effect (i.e. discordant LTA and PFA-100) (Figure 3). Of the 18 patients demonstrating discordant aspirin results, 14 (78\%) showed an exaggerated response to LD Epi (range 19-39\% aggregation), while only 4 (22\%) showed an appropriate response to LD Epi ( $\leq 18 \%$ aggregation). In contrast, only 4 of 11 (36\%) patients with concordant aspirin results by LTA and PFA-100 demonstrated abnormal aggregation in response to LD Epi, and the degree of abnormality was minimal (19-21\% aggregation versus $\leq 18 \%$ for an appropriate response). Therefore, an exaggerated response to LD Epi was more often seen when the LTA and PFA100 were discordant with regard to aspirin effect than when there was concordance between LTA and PFA-100.

\section{Discussion}

In an effort to aid in the characterization of hyperaggregable platelets, the aim of this study was to use LTA to identify a distinct pattern of platelet aggregation in patients who have experienced thrombosis without a significant family or personal history of thrombophilia. An abnormal, exaggerated response to LD Epi was found in $68 \%$ of patients with thrombosis compared to $36 \%$ of healthy controls $(\mathrm{P}=0.034)$. After exclusion of patients with either ASA or Plavix effect on LTA, an exaggerated response to LD Epi was found in $69 \%$ of cases. This may represent a distinct LTA pattern that can be used to identify patients with hyperaggregable platelets who may be at increased risk of experiencing thrombosis.

Few prior published studies have described a congenital platelet abnormality, termed sticky platelet syndrome, which is characterized by hyperaggregability with varying concentrations of ADP and/or Epi. ${ }^{1-4}$ It has been proposed that SPS is inherited in an autosomal dominant pattern ${ }^{1,3}$ and has a prevalence of 12 $33 \%$ in unexplained arterial thromboses and approximately $14 \%$ in unexplained venous

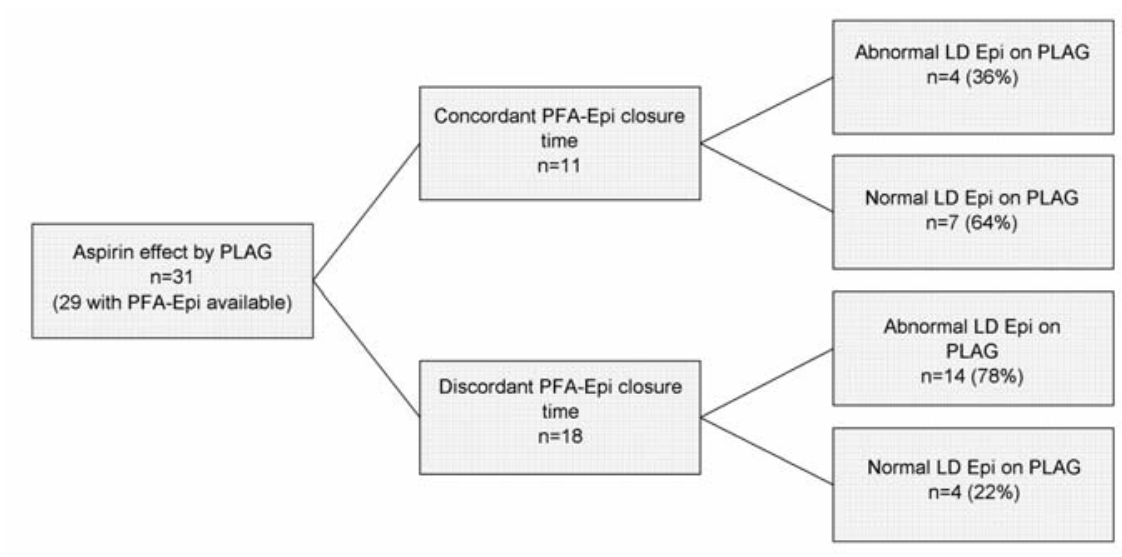

Figure 3. PFA-epinephrine results in patients with thrombosis who show aspirin effect by platelet aggregometry.

thromboses. ${ }^{2}$ Clinical symptoms associated with this syndrome are thought to include myocardial infarctions, transient ischemic attacks, optic neuropathy and recurrent venous thromboses. ${ }^{1,3}$ Arterial events are thought to occur more frequently than venous events. $^{2-4}$ Interestingly, in our study, although the presence of an exaggerated response to LD Epi occurred at relatively the same rate in patients with venous and arterial thromboses, venous thrombotic events occurred more commonly than arterial events. However, this may simply represent a selection bias for ordering LTA on patients with venous events over patients with arterial events.

\section{Platelet aggregometry}

Our study demonstrates that patients with thrombosis show exaggerated aggregation in response to LD Epi. This finding remains true even after patients with ASA or Plavix effect have been excluded. While studies have shown increased platelet reactivity in response to $\mathrm{LD}$ Epi in healthy volunteers, ${ }^{8-10}$ follow-up data and assessment of thrombosis formation in this supposedly hyperaggregable population subset has not been well evaluated. Previous studies have also suggested the presence of globally hyperaggregable platelets in which individuals that show increased aggregation to LD Epi may also show increased aggregation to other agonists, such as ADP.-10 In our study, however, statistically significant abnormalities in response to LD ADP or spontaneous aggregation were not identified. This finding is similar to the previously reported Type II aggregation pattern in SPS. ${ }^{1,3,5-7}$ With regards to heritability of hyperaggregable or sticky platelets, although previous studies have suggested an autosomal dominant inheritance pattern for sticky platelet syndrome, ${ }^{1,2}$ in our study, per the
LTA questionnaire, there was no significant family history of thrombosis. LTA results for patient family members were not available for review.

\section{Role of epinephrine}

Epinephrine is known to play a major role in thrombogenesis. Epi has been found to activate human platelets through alpha 2-adrenergic receptors. ${ }^{12}$ Epi itself can induce fibrinogen receptor exposure, as well as fibrinogen binding and platelet aggregation, in the absence of other agonists, including ADP, serotonin, platelet activating factor and thrombin. ${ }^{13}$ The roles of epinephrine and fibrinogen are also demonstrated in a murine study showing that P-selectin glycoprotein ligand-1 knock-out mice developed a lower number of thrombi following collagen/Epi injections as compared to wild-type mice. ${ }^{14}$ Plasma fibrinogen levels appear to regulate P-selectin expression on platelet membranes. ${ }^{15}$ Additionally, Epi has been found to interact with elevated shear stress in stenosed vessels to induce platelet aggregation. Wagner et al suggest that although this response requires functional GpIIb/IIIa complexes necessary for fibrinogen mediated aggregation, it is independent of vWF-Gplb mediated adhesion. ${ }^{16}$ It may be that the exaggerated response to LD Epi observed in patients with thrombosis is due to increased Epi receptor expression on the platelet surface or a gain of function mutation in platelets that renders platelets hyperreactive to lower in vivo concentrations of Epi.

\section{Aspirin effect and PFA-100}

LTA can be used to assess for the presence of aspirin effect in the form of a diminished response to AA. ${ }^{11}$ Additionally, aspirin effect has been demonstrated by a prolonged closure 
time with PFA-Epi. ${ }^{11,17}$ In the current study, however, PFA-Epi closure time was normal in a large proportion (62\%) of patients who showed aspirin effect by LTA, thus showing a discrepancy between the two assays. LTA is the gold standard for assessing platelet function by evaluating aggregation response to single agonists. Since PFA-100 measures global platelet function, it may be detecting platelet activation through receptors of pathways not inhibited by ASA and therefore, platelets are still functional despite an aspirin effect that is detectable by LTA. Prior studies have identified several factors affecting aspirin measurement by PFA100 , including elevated levels of fibrinogen, ${ }^{18}$ $\mathrm{VWF},{ }^{19-21}$ and adiponectin, ${ }^{22}$ as well as the presence of type 2 diabetes mellitus. ${ }^{22,23}$ Due to the retrospective nature of this project, these studies were not available for evaluation in our patient population.

In this study, the majority of the patients who showed normal PFA-Epi closure time despite the LTA showing aspirin effect also showed an exaggerated response to LD Epi by LTA. In contrast, an exaggerated response to LD Epi was seen in very few patients who showed concordant aspirin effect by both LTA and PFA-Epi. This may suggest, therefore, that in the discordant group, although aspirin effect is present by LTA, the expected prolongation of PFA-Epi is masked by platelet hyperaggregability, as demonstrated by exaggerated aggregation to LD Epi. We propose, therefore, that for a patient to be properly evaluated for aspirin effect, a LTA assessing response to AA and response to LD Epi be performed since the latter agonist may unmask an exaggerated platelet response that may require additional antiplatelet therapy.

\section{Limitations}

Since this study was a retrospective analysis, it is difficult to determine the exact timing of thrombosis in relation to LTA analysis; however, the majority of patients presented as outpatients and LTA was performed at least a few weeks after the acute thrombotic event. Prospective studies following both patients and healthy volunteers with normal and abnormal responses to LD Epi or LD ADP to assess for the development of thrombosis are needed to determine the significance of abnormal response to LD agonists and also to establish the threshold to call abnormal.

LTA agonist doses are not consistent between institutions and large control studies are needed to determine standard agonist doses according to the methods used (lighttransmittance $v s$ whole blood impedance aggregometry), as well as a standard assay duration (5 minutes vs 10 minutes). Interestingly, although our institution uses higher agonist doses than reported in other studies, ${ }^{5}$ it is our experience that the majority of normal cases do not show increased aggregation to our LD Epi concentration and the difference in LD Epi aggregation between study and control populations is still observed. We feel that because the overall pattern of hyperaggregability is maintained, our results are comparable to prior studies, despite the difference in agonist doses. This situation stresses the need for standardization of agonist doses between institutions.

We were only able to evaluate patients that were referred to us. The studied patient population consisted of approximately $1 / 3$ arterial events and $2 / 3$ venous events. Although the sampled population demonstrates an apparent dominance of venous events, the rate of platelet hyperaggregability was similar in both groups.

Although it is not realistic to request cessation of antiplatelet drugs in the presence of a recent thromboembolic event, ideally, it is best to test patients for hyperaggregable platelets when antiplatelet medication has been stopped for at least 7-10 days. Although many of the patients in this study reported recent antiplatelet medication use, the rate of platelet hyperaggregability remained unchanged following exclusion of all cases that showed evidence of ASA or Plavix use on LTA. Interestingly, despite this apparent limitation, hyperaggregability was also identified in the presence of antiplatelet medications.

\section{Conclusions}

Patients with thrombosis, either arterial or venous, demonstrated an exaggerated response to LD Epi more frequently than healthy controls. These patients reported no family history of thromboembolic events yet demonstrated hyperaggregable platelets. Therefore, platelet hyperreactivity is not necessarily a strictly hereditary condition, as previously described. In the presence of aspirin effect by LTA, a discordantly normal PFA-Epi closure time was associated with an exaggerated response to LD Epi. Normal PFA-100 results do not exclude ASA effect, which can be masked by underlying hyperaggregable platelets. Therefore, these data imply that there may be utility in using LTA to identify patients with a distinct hyperaggregable platelet phenotype who may be at increased risk of experiencing thrombosis and may benefit from a higher aspirin dose or the addition of a non-aspirin antiplatelet medication.

The etiology of an exaggerated response to LD Epi on LTA is still unknown. Some studies suggest a link between stressful situations and thrombosis, ${ }^{7,24}$ which may be related to increasing levels of Epi. Because Epi can induce fibrinogen receptor exposure, as well as facilitate fibrinogen binding and platelet aggregation, ${ }^{25}$ logic speculates that abnormal responses to LD Epi correlate with hyperresponsiveness to Epi and subsequent platelet hyperaggregability. Therefore, if there is an increased response to $\mathrm{LD} \mathrm{Epi,} \mathrm{there} \mathrm{is}$ increased fibrinogen receptor exposure, increased platelet aggregation and consequently, hyperaggregable platelets.

Due to the likely multifactorial nature of platelet hyperaggregability, a functional assay, such as LTA, is superior to genetic testing and can be used for both diagnostic and management purposes in patients with unexplained thromboses.

\section{References}

1. Mammen EF. Ten years' experience with the sticky platelet syndrome. Clin Appl Thromb Hemost 1995;1:66-72.

2. Bick RL. Sticky platelet syndrome: a common cause of unexplained arterial and venous thrombosis. Clin Appl Thromb Hemost 1998;4:77-81.

3. Mammen EF, Barnhart MI, Selik NR, et al. Sticky platelet syndrome: a congenital platelet abnormality predisposing to thrombosis? Folia Haematol Int Mag Klin Morphol Blutforsch 1988;115:361-5.

4. Frenkel EP, Mammen EF. Sticky platelet syndrome and thrombocythemia. Hematol Oncol Clin North Am 2003;1:63-83.

5. Kubisz P, Stasko J, Holly P. Sticky platelet syndrome. Semin Thromb Hemost 2013; 39:674-683.

6. Rubenfire M, Blevins RD, Barnhart M, et al. Platelet hyperaggregability in patients with chest pain and angiographically normal coronary arteries. Am J Cardiol 1986;57:657-60.

7. Mammen EF. Sticky platelet syndrome. Semin Thromb Hemost 1999;25:361-5.

8. Yee DL, Sun CW, Bergeron AL, et al. Aggregometry detects platelet hyperreactivity in healthy individuals. Blood 2005;106:2723-9.

9. Berger JS, Becker RC, Kuhn C, et al. Hyperreactive platelet phenotypes: Relationship to altered serotonin transporter number, transport kinetics and intrinsic response to adrenergic co-stimulation. Thromb Haemost 2013;109:85-92.

10. Yee DL, Bergeron AL, Sun CW, et al. Platelet hyperreactivity generalizes to multiple forms of stimulation. J Thromb Haemost 2006;4:2043-50.

11. Gurbel PA, Bliden KP, DiChiara J, et al. Evaluation of dose-related effects of aspirin on platelet function: results from the Aspirin-Induced Platelet Effect (ASPECT) study. Circulation 2007:3156-64. 
12. Lanza F, Cazenave JP, Beretz A, et al. Potentiation by adrenaline of human platelet activation and the inhibition by the alpha-adrenergic antagonist nicergoline of platelet adhesion, secretion and aggregation. Agents Actions 1986;18:58695.

13. Shattil SJ, Budzynski A, Scrutton MC. Epinephrine induces platelet fibrinogen receptor expression, fibrinogen binding, and aggregation in whole-blood in the absence of other excitatory agonists. Blood 1989;73:150-8.

14. Miszti-Blasius K, Debreceni IB, Felszeghy $\mathrm{S}$, et al. Lack of P-selectin glycoprotein ligand-1 protects mice from thrombosis after collagen/epinephrine challenge. Thromb Res 2011;127:228-34.

15. Yang H, Lang S, Zhai Z, et al. Fibrinogen is required for maintenance of platelet intracellular and cell-surface P-selectin expression. Blood 2009;114:425-36.

16. Wagner CT, Kroll MH, Chow TW, et al. Epinephrine and shear stress synergisti- cally induce platelet aggregation via a mechanism that partially bypasses VWFGP IB interactions. Biorheology 1996;33:209-29.

17. Reny JL, De Moerloose P, Dauzat M, Fontana P. Use of the PFA-100 closure time to predict cardiovascular events in aspirintreated cardiovascular patients: a systematic review and meta-analysis. J Thromb Haemost 2008:444-50.

18. Feher G, Koltai K, Papp E, et al. Aspirin resistance: possible roles of cardiovascular risk factors, previous disease history, concomitant medications and haemorrheological variables. Drugs Aging 2006;23:55967.

19. Favaloro EJ. Clinical utility of the PFA-100. Semin Thromb Hemost 2008;34:709-33.

20. Cattaneo M. Aspirin and clopidogrel: efficacy, safety, and the issue of drug resistance. Arterioscler Thromb Vasc Biol 2004;24:1980-7.

21. Faraday N, Becker DM, Yanek LR, et al. Relation between atherosclerosis risk fac- tors and aspirin resistance in a primary prevention population. Am J Cardiol 2006;98:774-9.

22. Takahashi S, Ushida M, Komine R, et al. Increased basal platelet activity, plasma adiponectin levels, and diabetes mellitus are associated with poor platelet responsiveness to in vitro effect of aspirin. Thromb Res 2007;119:517-24.

23. Crescente M, Di Castelnuovo A, Iacoviello L, et al. Response variability to aspirin as assessed by the platelet function analyzer (PFA)-100. A systematic review. Thromb Haemost 2008;99:14-26.

24. Chittoor SR. Sticky platelet syndrome: A case report and review of the literature. Clin Appl Thromb Hemost 1998;4:280-4.

25. Kunicki TJ, Nugent DJ. The influence of platelet glycoprotein polymorphisms on receptor function and risk for thrombosis. Vox Sanguinis 2002;83:85-90. 